\title{
Seven Criteria for Integer Sequences Being Graphic
}

\author{
Gerard Sierksma \\ DEPARTMENT OF ECONOMETRICS, \\ SECTION OPERATIONS RESEARCH \\ UNIVERSITY OF GRONINGEN, NETHERLANDS \\ Han Hoogeveen \\ CENTRE FOR MATHEMATICS AND \\ COMPUTER SCIENCE \\ AMSTERDAM, NETHERLANDS
}

\section{ABSTRACT}

Seven criteria for integer sequences being graphic are listed. Being graphic means that there is a simple graph with the given integer sequence as degree sequence. One of the criteria leads to a new and constructive proof of the well-known criterion of Erdös-Gallai.

\section{INTRODUCTION}

Let $\left(d_{1}, \ldots, d_{n}\right)$ be a nonincreasing sequence of positive integers with even sum. The sequence $\left(d_{1}, \ldots, d_{n}\right)$ is called graphic iff there is a simple graph (without loops and multiple edges) that has $\left(d_{1}, \ldots d_{n}\right)$ as degree sequence. In this paper seven criteria for such an integer sequence being graphic are listed; one of these is well known and due to Erdös and Gallai [5]. Proofs of the Erdös-Gallai Criterion can be found in Berge [1] and in Harary [10]. Harary's proof is rather lengthy and Berge's proof uses flows in networks. Recently, Choudum [4] has given a different proof which, in our opinion, is not very appealing either. Using the recently discovered Hässelbarth Criterion we are able to give a new and elegant proof.

\section{THE SEVEN CRITERIA}

In Theorem 1 it will be shown that the following conditions $(A)-(G)$ are all equivalent to " $\left(d_{1}, \ldots d_{n}\right)$ is graphic." 
A. The Ryser Criterion. See Bondy and Murty [3] and Ryser [12]. A sequence $\left(a_{1}, \ldots, a_{p} ; b_{1}, \ldots, b_{n}\right)$ is called bipartite-graphic iff there is a simple bipartite graph such that one component has degree sequence $\left(a_{1}, \ldots, a_{p}\right)$ and the other one has $\left(b_{1}, \ldots, b_{n}\right)$. Define $f=\max \left\{i \mid d_{i} \geq i\right\}$ and $\tilde{d}_{i}=$ $d_{i}+1$ if $i \in\langle f\rangle(=\{1, \ldots, f\})$ and $d_{i}=d_{i}$ otherwise. The criterion can be stated as follows:

The integer sequence $\left(\tilde{d}_{1}, \ldots, \tilde{d}_{n} ; \tilde{d}_{1}, \ldots, \tilde{d}_{n}\right)$ is bipartite-graphic.

B. The Berge Criterion. See, e.g., Berge [1]. Define $\left(\bar{d}_{1}, \ldots, \bar{d}_{n}\right)$ as follows: For $i \in\langle n\rangle, \bar{d}_{i}$ is the $i$ th column sum of the $(0,1)$-matrix, which has for each $k$ the $d_{k}$ leading terms in row $k$ equal to 1 except for the $(k, k)$ th term that is 0 and also the remaining entries are 0 . For example, if $d_{1}=3, d_{2}=2$, $d_{3}=2, d_{4}=2, d_{5}=1$, then $\bar{d}_{1}=4, \bar{d}_{2}=3, \bar{d}_{3}=2, \bar{d}_{4}=1, \bar{d}_{5}=0$, and the $(0,1)$-matrix becomes

$$
\left[\begin{array}{lllll}
0 & 1 & 1 & 1 & 0 \\
1 & 0 & 1 & 0 & 0 \\
1 & 1 & 0 & 0 & 0 \\
1 & 1 & 0 & 0 & 0 \\
1 & 0 & 0 & 0 & 0
\end{array}\right] .
$$

The criterion is

$$
\sum_{i=1}^{k} d_{i} \leq \sum_{i=1}^{k} d_{i} \text { for each } k \in\langle n\rangle
$$

C. The Erdös-Gallai Criterion. See, e.g., Bondy and Murty [3].

$$
\sum_{i=1}^{k} d_{i} \leq(k)(k-1)+\sum_{j=k+1}^{n} \min \left\{k, d_{j}\right\} \text { for each } k \in\langle n\rangle .
$$

D. The Fulkerson-Hoffman-McAndrew Criterion. See [5] and Grünbaum [6].

$$
\begin{array}{r}
\sum_{i=1}^{k} d_{i} \leq(k)(n-m-1)+\sum_{i=n-m+1}^{n} d_{i} \text { for each } k \in\langle n\rangle, \\
m \geq 0 \text { and } k+m \leq n .
\end{array}
$$

E. The Bollobás Criterion. See [2].

$$
\sum_{i=1}^{k} d_{i} \leq \sum_{i=k+1}^{n} d_{i}+\sum_{i=1}^{k} \min \left\{d_{i}, k-1\right\} \quad \text { for each } k \in\langle n\rangle
$$


F. The Grünbaum Criterion. See Grünbaum [7].

$$
\sum_{i=1}^{k} \max \left\{k-1, d_{i}\right\} \leq(k)(k-1)+\sum_{i=k+1}^{n} d_{i} \text { for each } k \in\langle n\rangle .
$$

G. The Hässelbarth Criterion. See Hässelbarth [11]. Define $\left(d_{1}^{*}, \ldots, d_{n}^{*}\right)$ as follows: For $i \in\langle n\rangle, d_{i}^{*}$ is the $i$ th column sum of the $(0,1)$-matrix in which the $d_{i}$ leading terms in row $i$ are 1's, and the remaining entries are 0 's. The criterion is

$$
\sum_{i=1}^{k} d_{i} \leq \sum_{i=1}^{k}\left(d_{i}^{*}-1\right) \quad \text { for each } k \in\langle f\rangle
$$

with $f=\max \left\{i \mid d_{i} \geq i\right\}$.

We call (G) the Hässelbarth Criterion because it was first described in Hässelbarth [11]. However, the form in which it is described in [11] is rather hidden, and there is also no proof. We will show that $(G)$ is in fact equivalent to criteria $(\mathrm{A})-(\mathrm{F})$. Hässelbarth's Criterion is concise and clear, and gives rise to an elegant proof of the Erdös-Gallai Criterion.

\section{THE MAIN THEOREM}

Theorem 1. Let $\left(d_{i}, \ldots d_{n}\right)$ be a positive integer sequence with even sum. Then the following holds:

Each of the criteria $(A)-(G)$ is equivalent to the statement that $\left(d_{1}, \ldots d_{n}\right)$ is graphic.

Proof. To prove the theorem we go through the following implications cycle:

(Graphic) $\stackrel{1}{\Rightarrow}(A) \stackrel{2}{\Rightarrow}(B) \stackrel{3}{\Rightarrow}(C) \stackrel{4}{\Rightarrow}(D) \stackrel{5}{\Rightarrow}(E) \stackrel{6}{\Rightarrow}(F) \stackrel{7}{\Rightarrow}(G) \stackrel{8}{\Rightarrow}$ (Graphic) .

1. Let $G=(V, E)$ be the simple graph that realizes $\left(d_{1}, \ldots, d_{n}\right)$. Starting with $G$, it is possible to construct a bipartite graph $B(G)$ with components $V_{1}$ and $V_{2}$ where $V_{1}=V_{2}(=V)$, and edge set $F$ defined as follows: If the edge joining $i$ and $j$ with $i, j \in V$ is in $E$, the edges joining $i \in V_{1}$ and $j \in V_{2}$ as well as the one joining $j \in V_{1}$ and $i \in V_{2}$ are in $F$. Moreover, the edges joining $i \in V_{1}$ and $j \in V_{2}$ for $i \in\langle f\rangle$ are also taken in $F$. For example, 
G

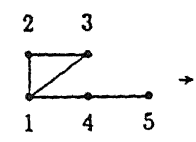

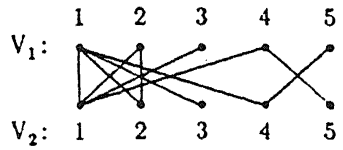

$B(G)$

The bipartite graph $B(G)$ has in both components degree sequence $\left(d_{1}+1, \ldots, d_{f}+1, d_{f+1}, \ldots, d_{n}\right)$. So the Ryser Criterion holds.

2. Let the sequence $\left(\tilde{d}_{1}, \ldots, \tilde{d}_{n} ; \tilde{d}_{1}, \ldots, \tilde{d}_{n}\right)$, as defined under $\mathrm{A}$, be bipartite-graphic. By a well-known theorem of Ryser (see, e.g., Bondy and Murty [3], p. 210, and Ryser [12]), it follows that

$$
\sum_{i=1}^{k} \tilde{d}_{i} \leq \sum_{i=1}^{k} \tilde{d}_{i}^{*} \text { for each } k \in\langle n\rangle,
$$

where $d_{i}^{*}$ is defined as in $(G)$. Hence $\tilde{d}_{i}^{*}=\bar{d}_{i}+1$ for each $i \in\langle f\rangle$, and $\tilde{d}_{i}=\bar{d}_{i}$ for each $i \in\langle n\rangle\langle\langle f\rangle$.

Moreover, $\tilde{d}_{i}=d_{i}+1$ for each $i \in\langle f\rangle$, and $\tilde{d}_{i}=d_{i}$ for each $i \in$ $\langle n\rangle\rangle\langle f\rangle$. If $k \leq f$, we find

$$
\sum_{i=1}^{k}\left(d_{i}+1\right)=\sum_{i=1}^{k} \tilde{d}_{i} \leq \sum_{i=1}^{k} \tilde{d}_{i}^{*}=\sum_{i=1}^{k}\left(\bar{d}_{i}+1\right),
$$

hence $\sum_{i=1}^{k} d_{i} \leq \sum_{i=1}^{k} \bar{d}_{i}$.

If $k \geq f+1$ we find

$$
\sum_{i=1}^{k} d_{i}+f=\sum_{i=1}^{k} \tilde{d}_{i} \leq \sum_{i=1}^{k} \tilde{d}_{i}^{*}=\sum_{i=1}^{k} \bar{d}_{i}+f, \quad \text { so that } \sum_{i=1}^{k} d_{i} \leq \sum_{i=1}^{k} \bar{d}_{i}
$$

Therefore, $\sum_{i=1}^{k} d_{i} \leq \sum_{i=1}^{k} \bar{d}_{i}$ for each $k \in\langle n\rangle$.

3. Consider the $(0,1)$-matrix corresponding to $\left(\bar{d}_{1}, \ldots, \bar{d}_{n}\right)$ as defined in (B). Take any $k \in\langle n\rangle$. Then $\sum_{i=1}^{k} \bar{d}_{i}$ is the number of 1 's in the first $k$ columns. In this $(0,1)$-matrix all diagonal elements are 0 , which means that in the submatrix consisting of the first $k$ rows and columns at most $k^{2}-k$ entries are 1 . On the other hand, each row $j$ has precisely $\min \left\{k, d_{j}\right\}$ l's on the first $k$ positions. Hence, for each $k \in\langle n\rangle$, we find

$$
\sum_{i=1}^{k} d_{i} \leq \sum_{i=1}^{k} \bar{d}_{i} \leq(k)(k-1)+\sum_{j=k+1}^{n} \min \left\{k, d_{j}\right\} .
$$

4. For each $k \in\langle n\rangle, m \geq 0$ and $n-m \geq k$, it follows that

$$
\begin{aligned}
\sum_{i=1}^{k} d_{i} & \leq(k)(k-1)+\sum_{j=k+1}^{n} \min \left\{k, d_{j}\right\} \\
& \leq(k)(k-1)+\sum_{j=k+1}^{n-m} \min \left\{k, d_{j}\right\}+\sum_{j=n-m}^{n} \min \left\{k, d_{j}\right\}
\end{aligned}
$$




$$
\begin{aligned}
& \leq(k)(k-1)+(k)(n-m-k)+\sum_{j=n-m+1}^{n} \min \left\{k, d_{j}\right\} \\
& \leq(k)(n-m-1)+\sum_{j=n-m+1}^{n} d_{j} .
\end{aligned}
$$

5. Take any $k \in\langle n\rangle$. If $d_{1}, \ldots, d_{k} \leq k-1$, then substitute $n-m=k$, and we are done. So, we may assume that $d_{1}, \ldots, d_{l} \geq k-1$ and $d_{l+1}, \ldots, d_{k}<k-1$ for some $l<k$. Then the following holds:

$$
\begin{aligned}
\sum_{i=1}^{k} d_{i} & =\sum_{i=1}^{l}+\sum_{i=l+1}^{k} d_{i} \leq \text { (using (D)) } \\
& \left.\leq(l)(n-m-1)+\sum_{i=n-m+1}^{n} d_{i}+\sum_{i=l+1}^{k} d_{i}=\text { (using } m=n-k\right) \\
& =(l)(k-1)+\sum_{i=k+1}^{n} d_{i}+\sum_{i=l+1}^{k} d_{i} \\
& =\sum_{i=k+1}^{n} d_{i}+\sum_{i=1}^{l}(k-1)+\sum_{i=l+1}^{n} d_{i} \\
& =\sum_{i=k+1}^{n} d_{i}+\sum_{i=1}^{l} \min \left\{d_{i}, k-1\right\}+\sum_{i=l+1}^{k} \min \left\{d_{i}, k-1\right\} \\
& =\sum_{i=k+1}^{n} d_{i}+\sum_{i=1}^{k} \min \left\{d_{i}, k-1\right\} .
\end{aligned}
$$

6. For each $k \in\langle n\rangle$ the following holds:

$$
\begin{aligned}
\sum_{i=1}^{k} d_{i} & \leq \sum_{i=k+1}^{n} d_{i}+\sum_{i=1}^{k} \min \left\{d_{i}, k-1\right\} \\
& \Leftrightarrow \sum_{i=1}^{k} d_{i}+\sum_{i=1}^{k} \max \left\{-d_{i},-k+1\right\} \leq \sum_{i=k+1}^{n} d_{i} \\
& \Leftrightarrow \sum_{i=1}^{k} \max \left\{0, d_{i}-k+1\right\} \leq \sum_{i=k+1}^{n} d_{i} \\
& \Leftrightarrow \sum_{i=1}^{k}\left[\max \left\{k-1, d_{i}\right\}-k+1\right] \leq \sum_{i=k+1}^{n} d_{i} \\
& \Leftrightarrow \sum_{i=1}^{k} \max \left\{k-1, d_{i}\right\} \leq(k)(k-1)+\sum_{i=k+1}^{n} d_{i} .
\end{aligned}
$$

7. Suppose to the contrary that there is an index $k \in\langle f\rangle$ such that

$$
\sum_{i=1}^{k} d_{i}>\sum_{i=1}^{k}\left(d_{i}^{*}-1\right)
$$


Note that $f \leq n-1$. If $f=n-1$, then $d_{i}=n-1$ for each $i \in\langle n\rangle$ and $(G)$ is trivially true. So we may assume that $f \leq n-2$. There now exists an index $m \in\{k, \ldots, n\}$ such that $d_{i} \geq k$ for $i \leq m$ and $d_{i}<k$ for $i \geq m+1$.

We then have,

$$
\begin{aligned}
\sum_{i=1}^{k} d_{i} & >\sum_{i=1}^{k}\left(d_{i}^{*}-1\right)=(k)(m-1)+\sum_{i=m+1}^{n} d_{i} \geq(\operatorname{using}(\mathrm{G})) \\
& \geq(k)(m-1)-(m)(m-1)+\sum_{i=1}^{m} \max \left\{m-1, d_{i}\right\}
\end{aligned}
$$

Hence,

$$
\begin{aligned}
\sum_{i=1}^{m} \max \{m-1, & \left.d_{i}\right\}<(m-k)(m-1)+\sum_{i=1}^{k} d_{i} \\
= & \sum_{i=1}^{k} d_{i}+\sum_{i=k+1}^{m}(m-1)\left(\text { with } \sum_{i=m+1}^{m}(m-1) \text { defined } 0\right) \\
& \leq \sum_{i=1}^{k} \max \left\{m-1, d_{i}\right\}+\sum_{i=k+1}^{m} \max \left\{m-1, d_{i}\right\} \\
& =\sum_{i=1}^{m} \max \left\{m-1, d_{i}\right\} .
\end{aligned}
$$

This is a contradiction. So Hässelbarth's Criterion holds.

8. Using Hässelbarth's Criterion we finally show that the integer sequence $\left(d_{1}, \ldots, d_{n}\right)$ is graphic.

Define for each $i \in\langle f\rangle$

$$
a_{i}=d_{i}-i+1 \text { and } b_{i}=d_{i}^{*}-i
$$

Hässelbarth's Criterion can then be rewritten as follows:

$$
\sum_{i=1}^{k} a_{i} \leq \sum_{i=1}^{k} b_{i} \text { for each } k \in\langle f\rangle
$$

Our first objective is to transform the $(0,1)$-matrix $A$, as defined under (G), into a symmetric (0,1)-matrix $A^{\prime \prime}$ with 0 's on the main diagonal. Obviously, $A^{\prime \prime}$ corresponds with a simple graph. In general, however, this graph does not satisfy the original degree sequence $\left(d_{1}, \ldots, d_{n}\right)$. So we need a transformation that results in a symmetric matrix $A^{*}$ with row sum vector the original degree sequence. The first step, in obtaining $A^{\prime \prime}$, is the following algorithm; it results, generally, in a nonsymmetric matrix $A^{\prime}$. 
$k:=f$

WHILE $k>1$ DO

BEGIN

IF $a_{k}=b_{k}$ THEN $a_{k}^{\prime}=a_{k}$

IF $a_{k}<b_{k}$ THEN

BEGIN $a_{k}^{\prime}:=a_{k}+\left\lfloor\frac{1}{2}\left(b_{k}-a_{k}\right)\right\rfloor$

IF $b_{k}-a_{k}$ odd THEN $a_{k-1}:=a_{k-1}+1$

END

IF $a_{k}>b_{k}$ THEN

BEGIN $a_{k}^{\prime}:=b_{k}$ and $a_{k-1}:=a_{k-1}+a_{k}-b_{k}$ END

$$
k:=k-1
$$

END

$a_{1}^{\prime}:=a_{1}+\frac{1}{2}\left(b_{1}-a_{1}\right)$

Define $b_{i}^{\prime}=a_{i}^{\prime}$ for $i \in\langle f\rangle$

The above algorithm needs the following remarks:

(1) This step can be visualized as follows

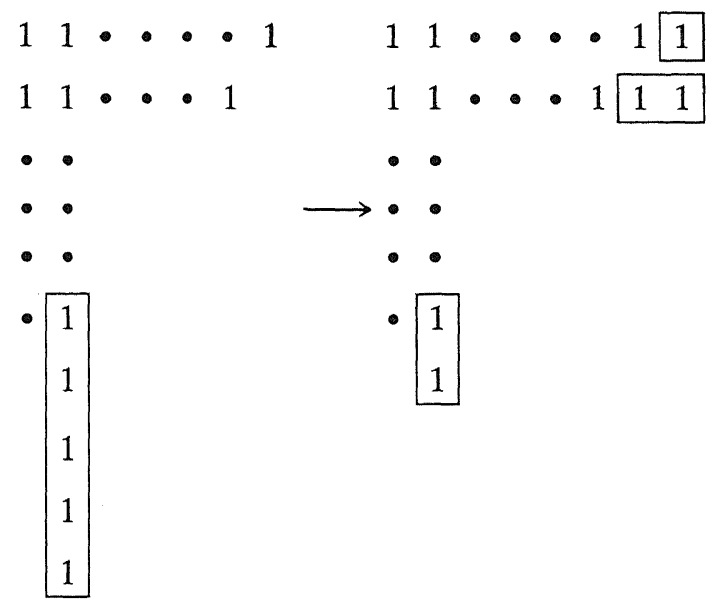

(2) In this step $a_{k}-b_{k}$ 1's from row $k$ are lifted to row $k-1$.

(3) The fact that $b_{1}-a_{1}$ (with $a_{1}$ the number of 1's in row 1 after applying the $f-1$ steps of the algorithm!) is nonnegative and even, is the key of the algorithm. The proof is as follows.

Suppose, there is a maximal index $\alpha \geq 2$ such that $b_{i} \leq a_{i}$ for each $i=2, \ldots, \alpha$. Hence, for the rows $\alpha, \alpha-1, \ldots, 2$ only loop (2) of the algorithm is used. Row $\alpha$ receives at most one 1 from column $\alpha+1$, namely 
in case $b_{\alpha+1}-a_{\alpha+1}$ is odd. Therefore, $a_{\alpha}:=a_{\alpha}(+1)$. The final step, when executing loop (2), implies that

$$
a_{1}:=\sum_{i=1}^{\alpha} a_{i}-\sum_{i=2}^{\alpha} b_{i}(+1),
$$

whereas the $a_{i}$ 's in the right-hand side of this expression are the original $a_{i}$ 's of the matrix $A$.

The Hässelbarth Criterion implies that this new $a_{1}$ is $\leq b_{1}$. This can be seen as follows.

In case $b_{\alpha+1}-a_{\alpha+1}$ is even, we do not have the extra +1 , hence, $a_{1} \leq b_{1}$ is nothing more than the Hässelbarth Criterion for $k=\alpha$. In case $b_{\alpha+1}-a_{\alpha+1}$ is odd, we have to show that $1+\sum_{i=1}^{\alpha} a_{i}-\sum_{i=2}^{\alpha} b_{i} \leq b_{1}$. Suppose, to the contrary, that $1+\sum_{i=1}^{\alpha} a_{i}-\sum_{i=2}^{\alpha} b_{i}>b_{1}$. As, on the other hand, $\sum_{i=1}^{\alpha} a_{i}-\sum_{i=2}^{\alpha} b_{i} \leq b_{1}$, it follows that $b_{1}=\sum_{i=1}^{\alpha} a_{i}-\sum_{i=2}^{\alpha} b_{i}$. But this implies that $1+\left[\sum_{i=1}^{\alpha} a_{i}-\sum_{i=2}^{\alpha} b_{i}\right]+b_{1}$ is not even, which contradicts the facts that the total number of 1 's is even and that $a_{2}^{\prime}=b_{2}^{\prime}, \ldots, a_{f}^{\prime}=b_{f}^{\prime}$. Hence, $a_{1} \leq b_{1}$.

If there does not exist such a smallest $\alpha$, then $b_{2} \geq a_{2}$ (with $a_{2}$ after applying $f-2$ times of the algorithm) and hence, $a_{1} \leq b_{1}$ (with $a_{1}$ after applying $f-1$ steps of the algorithm) according to the Hässelbarth Criterion.

Needless to say that $b_{1}-a_{1}$ (with $a_{1}$ after applying $f-1$ steps of the algorithm) is even, so that $a_{1}^{\prime}$ is well defined.

For $a_{i}^{\prime}>0, A^{\prime}$ has $a_{i}^{\prime}+i+1$ 1's in the first $a_{i}^{\prime}+i-1$ positions of row $i(i \in\langle f\rangle), b_{j}^{\prime}+j$ 's in the first $b_{j}^{\prime}+j$ positions in column $j(j \in\langle f\rangle)$, and zeros elsewhere.

The (0,1)-matrix $A^{\prime \prime}$ results now as follows: A number 1 on the main diagonal in row $i$ is replaced by the number 0 and the 0 following the 1 in position $a_{i}^{\prime}+i-1$ in row $i$ is replaced by the number $1 ; i \in\langle f\rangle$.

The so-formed matrix $A^{\prime \prime}=\left\{a_{i j}^{\prime \prime}\right\}$ is symmetric and has zeros on its main diagonal. Let the row sum vector of $A^{\prime \prime}$ be $\left(d_{1}^{\prime}, \ldots, d_{n}^{\prime}\right)$.

The matrix $A^{*}$ is now formed in the following way:

If $\left(d_{1}^{\prime}, \ldots, d_{n}^{\prime}\right) \neq\left(d_{1}, \ldots, d_{n}\right)$, then consider the sequence $d_{1}^{\prime}-d_{1}$, $\ldots, d_{n}^{\prime}-d_{n}$.

As loops (1) and (2) of the algorithm "lift" 1's to rows with lower indices, there is a greatest index $s$ and a smallest index $t$, such that

$$
d_{s}^{\prime} \geq d_{s}+1, \quad d_{t}^{\prime} \leq d_{t}-1, \text { and } s<t .
$$

As $d_{s}^{\prime} \geq d_{s}+1 \geq d_{t}+1 \geq d_{t}^{\prime}+2$, there is an index $k$ with $s \neq k \neq t$ and

$$
a_{s k}^{\prime \prime}=a_{k s}^{\prime \prime}=1, \quad a_{k t}^{\prime \prime}=a_{t k}^{\prime \prime}=0 .
$$

The matrix $A^{\prime \prime}$ is now changed into the matrix $\left\{a_{i j}^{*}\right\}$ satisfying $a_{i j}^{*}=a_{i j}^{\prime \prime}$ for each $i$ and $j$, except that

$$
a_{s k}^{*}=a_{k s}^{*}=0 \quad \text { and } \quad a_{k t}^{*}=a_{t k}^{*}=1 \text {; }
$$


i.e.,

\begin{tabular}{|c|c|c|c|c|c|c|c|}
\hline & $s$ & $k \quad t$ & $t$ & & $s$ & $k$ & $t$ \\
\hline$s$ & & 1 & & $s$ & & 0 & \\
\hline$k$ & 1 & & $0 \longrightarrow$ & $k$ & 0 & & 1 \\
\hline$t$ & & 0 & & $t$ & & 1 & \\
\hline
\end{tabular}

or for the corresponding graph,

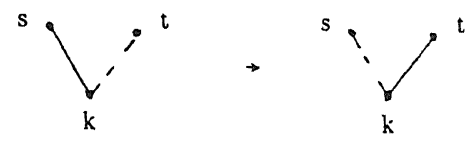

This procedure is repeated until the row sums of the resulting matrix $A^{*}$ are $d_{1}, \ldots, d_{n}$. Note that the number of steps in this procedure is equal to the number of 1's "lifted" by the algorithm that transforms $A$ into $A^{\prime}$. The graph corresponding to $A^{*}$ is a realization of the sequence $\left(d_{1}, \ldots, d_{n}\right)$. This completes the proof.

\section{References}

[1] C. Berge, Graphs and Hypergraphs. North Holland, Amsterdam, and American Elsevier, New York (1973).

[2] B. Bollobás, Extremal Graph Theory. Academic Press, New York (1978).

[3] J.A. Bondy and U.S.R. Murty, Graph Theory with Applications. American Elsevier, New York (1976).

[4] S. A. Choudum, A simple proof of the Erdös-Gallai theorem on graph sequences. Bull. Austr. Math. Soc. 33 (1986) 67-70.

[5] P. Erdös and T. Gallai, Graphs with given degree of vertices. Mat. Lapok 11 (1960) 264-274.

[6] D. R. Fulkerson, A. J. Hofman, and M. H. McAndrew, Some properties of graphs with multiple edges. Can. J. Math. 17 (1965) 166-177.

[7] B. Grünbaum, Graphs and complexes. Report of the University of Washington, Seattle, Math., 572B, 1969 (private communication).

[8] S. L. Hakimi, On realizability of a set of integers as degrees of the vertices of a linear graph I. J. Soc. Indust. Appl. Math. 10 (1962) 496-506.

[9] S. L. Hakimi, On realizability of a set of integers as degree of the vertices of a linear graph II. Uniqueness. J. Soc. Indust. Appl. Math. 11 (1963) 135-147.

[10] F. Harary, Graph Theory. Addison-Wesley, Reading, MA (1969).

[11] W. Hässelbarth, Die Verzweigtheit von Graphen. Match. 16 (1984) 3-17.

[12] H. J. Ryser, Combinatorial properties of matrices of zeros and ones. Can. J. Math. 9 (1957) 371-377. 